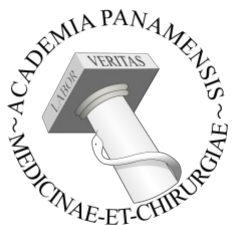

\title{
Artículos originales \\ El empoderamiento de la mujer panameña y su relación con la violencia conyugal según la Encuesta Nacional de Salud Sexual y Reproductiva
}

\author{
[Panamanian women's empowerment and its relationship with spousal violence \\ according to the National Survey on Sexual and Reproductive Health] \\ Haydeé Flores Castro, Ruth G. De León, Fermina Chamorro, Alba Mendoza, Ligia Martínez García \\ Departamento de Investigación en Salud Sexual y Reproductiva del Instituto Conmemorativo Gorgas de Estudios de la Salud.
}

Palabras Claves: violencia conyugal,violencia sexual, violencia física,empoderamiento femenino

Keywords: marital violence,physical violence,sexual violence,female empowerment

Correspondencia a:

Haydeé Flores Castro

Correo electrónico:

hflores@gorgas.gob.pa

Recibido:

1 de marzo de 2021

Publicado:

26 de octubre de 2021

Aspectos bioéticos:

La información cruda anonimizada se compartirá a solicitud por el autor corresponsal. Aprobado por el Comité de Bioética Institucional.

Financiamiento:

Este trabajo recibió fianciamiento total o parcial de: MEF. La información cruda anonimizada se compartirá a solicitud por el autor corresponsal.

\section{Resumen}

Introducción: La Organización Mundial de la Salud reconoce que la violencia conyugal es una problemática que va más allá del ámbito familiar, afecta a todos los sectores de la sociedad convirtiéndose en un grave problema de salud pública y de derechos humanos.

Objetivo: examinar la relación entre elementos del empoderamiento femenino y tipos de violencia conyugal. Métodos: con datos de la Encuesta Nacional de Salud Sexual y Reproductiva (2014-2015) se analizaron 3679 mujeres casadas/unidas. Se construyeron tres indicadores de empoderamiento como variable independiente, como variable dependiente se estudió la violencia física/sexual. Los datos fueron analizados con el programa SPSS utilizando descripciones de frecuencia, cruce de variables y pruebas de significancia estadística. Resultados: Aquellas mujeres que más justifican la violencia conyugal, son las que más sufren agresiones físicas como empujones y/o sacudidas (15\%), las que no justifican la violencia por ninguna razón, solo $4.1 \%$ sufren ataques de este tipo $(p=0.000)$. El $3.1 \%$ de las mujeres con menor participación en el hogar son las que menos agresiones sufren en comparación con las que toman más decisiones y sufren más agresiones $(8.1 \%)$ ( $p<$ 0.000). El grado de participación en aspectos importantes de Salud Sexual y Reproductiva (SSR), muestra que aquellas que no tenían ningún tipo de intervención, padecen mayores agresiones de tipo sexual (7.3\%), estas agresiones disminuyen cuando la mujer toma mayores decisiones en SSR $(0.4 \%)(p=0.000)$. Conclusiones: Hay una asociación positiva entre una menor vivencia de violencia física/sexual con mayores niveles de autonomía en temas de SSR y Rechazo a la Violencia Conyugal.

\begin{abstract}
Introduction: The World Health Organization recognizes that spousal violence is a problem that goes beyond the family sphere and affects all sectors of society, becoming a serious public health and human rights problem. Objective: to examine the relationship between elements of female empowerment and types of spousal violence. Methods: using data from the National Survey of Sexual and Reproductive Health (2014-2015), 3679 married/unmarried women were analyzed. Three indicators of empowerment were constructed as independent variable, as dependent variable physical/sexual violence was studied. The data were analyzed with the SPSS program using frequency descriptions, crossing of variables and statistical significance tests. Results: Those women who most justify spousal violence are those who suffer more physical aggressions such as pushing and/or shaking (15\%), those who do not justify violence for any reason, only $4.1 \%$ suffer attacks of this type $(p=0.000)$. The $3.1 \%$ of women with less participation in the home suffer the least aggressions compared to those who make more decisions and suffer more aggressions $(8.1 \%)(p<$ 0.000). The degree of participation in important aspects of Sexual and Reproductive Health $(\mathrm{SRH})$, shows that those who did not have any type of intervention, suffer greater aggressions of sexual type $(7.3 \%)$, these aggressions decrease when the woman makes greater decisions in SRH (0.4\%) $(p=0.000)$. Conclusions: There is a positive association between a lower experience of physical/sexual violence with higher levels of autonomy in SRH issues and Rejection of Spousal Violence.
\end{abstract}

Órgano oficial de la Academia Panameña de Medicina y Cirugía y del Instituto Commemorativo Gorgas para Estudios de la Salud (ICGES). Indexado en LILACS, EBSCO, y www.revistasmedicas.org.
R M P

RMDP 2021; 41(2):2-7 


\section{INTRODUCCIÓN}

En toda la historia del ser humano nunca antes habíamos tenido tanta conciencia de vivir en un mundo violento como hoy día. Esta visibilización de la violencia, en cualquiera de sus formas, puede deberse a un mayor acceso al conocimiento e información, en un mundo donde las personas al estar más interconectadas que nunca, son más conscientes de todos los fenómenos sociales en una amplia escala local y mundial.

En Panamá, al igual que muchos países, las cifras de violencia de género son alarmantes, solo en el año 2019 hubo un reporte de 16,851 denuncias registradas en el Ministerio Público a nivel nacional y los casos de femicidios llegaron a 21 víctimas, sin contar los intentos de femicidios [1]. Probablemente de todos los tipos de violencia de género, la más frecuente es la infligida por la pareja de la mujer. La Organización Mundial de la Salud (OMS), define la violencia conyugal como cualquier comportamiento, dentro de una relación íntima, que cause o pueda causar daño físico, psíquico o sexual a los miembros de la relación [2].

La violencia de género, tiene su génesis en la dominación y control vinculado a la postura dominante del hombre sobre la mujer. Son las desigualdades de poder que llevan a muchas mujeres a una posición de subordinación respecto al hombre. Históricamente las mujeres han tenido un limitado acceso y control de los recursos necesarios para dirigir sus propias vidas y su entorno. Este proceso que lleva a las mujeres a tomar un mayor control sobre los recursos intelectuales y materiales se define como empoderamiento femenino y permite desafiar la discriminación de género producto de una sociedad basada en la ideología del patriarcado [3].

Esto hace suponer que, aquellas mujeres que adquieren un mayor control de sus vidas tienen menos riesgos de ser víctimas de la violencia conyugal. Sin embargo, este proceso de empoderamiento femenino no siempre es fácil acceder a él sin importar el entorno en que viven las mujeres, así vemos muchas de ellas víctimas de la violencia marital independiente de sus entornos socioeconómicos, culturales o geográficos como indica un estudio multi-país de la OMS [4].

El objetivo de este trabajo es examinar la relación entre tres elementos del empoderamiento de las entrevistadas: Rechazo a la violencia conyugal, poder de decisión de la mujer en el hogar y poder de decisión de la mujer sobre su propia Salud Sexual y Reproductiva, con el riesgo que tiene la mujer de sufrir violencia física y sexual por parte de su pareja íntima.

\section{MATERIALES Y MÉTODOS} Este es un análisis secundario que utilizó datos de la En- cila entre 0 y 4 , donde el valor mínimo 0 refleja la ausencuesta Nacional de Salud Sexual y Reproductiva (ENAS- cia de participación de la mujer en estos aspectos.
SER), realizada en zonas urbanas, rurales e indígenas de la República de Panamá en los años 2014 a 2015. Esta encuesta generó información sobre indicadores claves de la sexualidad y la reproducción en mujeres. Los datos fueron recopilados a través de un cuestionario usando la técnica de la entrevista.

Participaron de la encuesta un total de 5,616 mujeres, de las cuales se incluyeron en este análisis a solo 3,679 que cumplían con los dos siguientes criterios: estar conviviendo con una pareja masculina estable (casada o en unión conyugal) al momento de la encuesta y mujeres que contaban con privacidad, al momento de responder las preguntas de violencia doméstica.

Consideraciones éticas: La ENASSER fue aprobada por el Comité de Bioética de la Investigación del Instituto Conmemorativo Gorgas de Estudios de la Salud y las mujeres consintieron en participar, luego de ser informadas de los objetivos de la encuesta, sus procedimientos y sus derechos como sujetos de investigación.

Para estudiar las variables independientes se construyeron tres indicadores de empoderamiento femenino:

Rechazo a la Violencia Conyugal: El primer indicador está basado en seis preguntas incluidas en la encuesta sobre si se "justifica que un esposo golpee a su esposa si": 1) ella sale de casa sin decírselo a su esposo, 2) ella descuida o desatiende a los niños, 3) ella discute con su esposo, 4) ella rehúsa tener relaciones sexuales con su esposo, 5) ella deja quemar la comida, 6) ella le es infiel a su esposo. Las puntuaciones oscilan entre 0 y 6 , donde los valores mayores a 2 corresponden a aquellas mujeres que más justifican la violencia conyugal.

Poder de Decisión de la Mujer en el Hogar: El segundo indicador se construyó en base a la capacidad que tiene la mujer en tomar decisiones de manera autónoma, es decir sin el permiso o autorización de su esposo. Para este indicador se tomaron tres preguntas de la encuesta sobre "quién toma la decisión en determinadas situaciones": 1) cómo gastar el dinero que la mujer gana, 2) compras importantes en el hogar, 3) visitar a familiares o parientes de la mujer. Este indicador oscila entre 0 y 3 , donde el valor mínimo 0 refleja la ausencia de decisiones autónomas en el hogar.

Poder de Decisión de la Mujer sobre su Salud Sexual y Reproductiva (SSR): El tercer indicador se construyó a partir del número de decisiones que toma la propia mujer de manera autónoma, sobre aspectos claves de su salud sexual y reproductiva. Se utilizaron cuatro preguntas sobre: 1) la búsqueda de atención médica, 2) el uso de un método anticoncepción, 3) la decisión de tener relaciones sexuales y d) la decisión de usar un condón. Su valor os- 
Flores y cols. - El empoderamiento de la mujer panameña.

Como variables dependientes se estudió la violencia físi- Esta tendencia se observa en todos los tipos de violencia ca y sexual a través de 10 ítems diferentes: que van des- física y sexual presentadas en esta tabla: aquellas mujede empujar o hamaquear, abofetear, doblar el brazo, res que más justifican la violencia conyugal son las que golpes con el puño, patear/arrastrar, intentar quemarla/ más agresiones enfrentan, a medida que la mujer da measfixiarla, agresión con chuchillos o armas de fuego, for- nos razones (de 1-2) para justificar la violencia o no la zarla a tener relaciones sexuales, forzarla físicamente a justifica por ningún motivo los porcentajes disminuyen. realizar cualquier acto sexual y forzarla con amenazas o Solo un tipo de agresión física no fue estadísticamente intimidación a realizar cualquier acto sexual.

Los datos fueron analizados con el programa estadístico significativa $(p=0.782)$ : cuando el ataque o amenaza involucró agresión con arma de fuego o arma blanca. Otro aspecto importante a resaltar es el hecho que cuando las SPSS versión 22 utilizando descripciones de frecuencia simple, cruce de variables y pruebas de significancia estadística. Para estudiar las diferencias entre las variables independientes y dependientes se utilizó la prueba de chi cuadrado estableciendo un nivel de significancia estadística mínima de menos de 0.05 .

\section{RESULTADOS}

agresiones pasan de ser empujones, sacudidas a eventos cada vez más violentos donde puede haber más señales o riesgos para la integridad físicas de la víctima, los porcentajes de justificación van disminuyendo.

La tabla 2 muestra relación entre el grado de participación de la mujer, en decisiones importantes en el hogar (Indicador 2: Poder de Decisión de la Mujer en el Hogar) y cómo este nivel de participación se asocia a la presencia o no de violencia física y/o sexual que sufre la entrevistada por parte de su pareja íntima.

En la tabla 1 se presentan los resultados del grado de justificación que la entrevista otorga a la violencia conyugal que puede sufrir cualquier mujer (Indicador 1: Rechazo a la Violencia Conyugal) y como este grado de justificación se asocia a la presencia o no de violencia física y/o sexual que la propia entrevistada ha sufrido por parte de su actual esposo o pareja.

El 15\% de las mujeres que más justifican la violencia conyugal (en tres o más razones) son las que sufren más agresiones en el primer renglón del cuadro (empujones o sacudidas), a medida que el número de razones va disminuyendo el porcentaje de agresión es menor: $5.7 \%$ justifican la violencia por una a dos razones y $4.1 \%$ de las mujeres que sufren agresión de este tipo, no justifican la violencia por ninguna razón $(p=0.000)$.

El $3.1 \%$ de las entrevistadas que no tienen ningún tipo de participación en los asuntos importantes en el hogar, son las que menos agresiones sufren como empujones o sacudidas, pero los porcentajes van aumentando en aquellas mujeres con mayor número de decisiones en el hogar: $4.7 \%$ en las que toman de 1 a 2 decisiones y $8.1 \%$ en aquellas que toman 3 ó más decisiones, estas diferencias son estadísticamente significativas. Este comportamiento se aprecia en todos los tipos de violencia física y sexual, donde las mujeres que tienen mayor número de decisiones en el hogar, son las que sufren mayores agresiones por parte de sus cónyuges, igualmente todas estas diferencias arrojan una p menor a 0.05 .

Finalmente se presenta la relación entre el grado de participación de la mujer, en aspectos claves de su propia sa-

Tabla 1. Relación entre las razones que da la entrevistada para justificar la violencia física conyugal y los tipos de agresión física/sexual que sufre la entrevistada por parte de su pareja

Tipos de agresión física/sexual que sufre la entrevistada por parte de su pareja
Número de razones que da la entrevistada para justificar la violencia física conyugal \begin{tabular}{l|l} 
Mujeres que & Mujeres que
\end{tabular} justifican la no justifica la violencia en 3 o $\quad$ violencia en 1 a violencia por más razones 2 razones ningún motivo

\begin{tabular}{|c|c|c|c|}
\hline & & & \\
\hline Empujó/hamaqueó & 15.0 & 5.7 & 4.1 \\
\hline La abofeteó & 10.3 & 4.0 & 2.6 \\
\hline Le dobló el brazo o le haló el cabello & 12.2 & 4.3 & 2.0 \\
\hline Le pegó con el puño o con algún objeto que le pudo lastimar & 8.0 & 3.9 & 3.1 \\
\hline La pateó, arrastró o le dio palizas & 6.5 & 2.0 & 0.5 \\
\hline Intentó quemarla o asfixiarla & 3.0 & 1.5 & 0.4 \\
\hline La atacó o amenazó con un cuchillo, pistola u otra arma & 1.3 & 0.6 & 0.7 \\
\hline La forzó físicamente a tener relaciones sexuales & 13.1 & 3.1 & 1.9 \\
\hline La forzó físicamente a realizar cualquier acto sexual que ella no quería & 7.0 & 1.0 & 0.6 \\
\hline La forzó con amenazas a realizar actos sexuales que ella no quiere & 5.0 & 0.7 & 0.5 \\
\hline
\end{tabular}

Fuentes: preparado por los autores con datos de ENASSER 2014-2015. 
Tabla 2. Relación entre la toma de decisiones en el hogar y los tipos de violencia física/

sexual que sufre la entrevistada por parte del esposo

Tipos de agresión física/sexual que sufre la entrevistada por parte de su pareja

Número de decisiones en el hogar en que la entrevistada participa

\begin{tabular}{|c|c|c|c|}
\hline de su pareja & $\begin{array}{l}\text { Mujeres que no } \\
\text { participan de } \\
\text { ninguna decisión }\end{array}$ & $\begin{array}{c}\text { Mujeres que } \\
\text { participan de } 1 \text { a } 2 \\
\text { decisiones }\end{array}$ & $\begin{array}{c}\text { Mujeres que } \\
\text { participan en } 3 \text { ó } \\
\text { más decisiones }\end{array}$ \\
\hline Empujó/hamaqueó & 3.1 & 4.7 & 8.1 \\
\hline La abofeteó & 2.1 & 3.3 & 4.1 \\
\hline Le dobló el brazo o le haló el cabello & 2.0 & 2.5 & 4.7 \\
\hline Le pegó con el puño o con algún objeto que le pudo lastimar & 1.7 & 3.0 & 3.7 \\
\hline La pateó, arrastró o le dio palizas & 0.6 & 0.8 & 1.6 \\
\hline Intentó quemarla o asfixiarla & 0.8 & 2.0 & 3.5 \\
\hline La atacó o amenazó con un cuchillo, pistola u otra arma & 0.6 & 0.7 & 1.6 \\
\hline La forzó físicamente a tener relaciones sexuales & 1.7 & 2.5 & 3.7 \\
\hline $\begin{array}{l}\text { La forzó físicamente a realizar cualquier acto sexual que ella no } \\
\text { quería }\end{array}$ & 0.4 & 0.8 & 1.5 \\
\hline La forzó con amenazas a realizar actos sexuales que ella no quiere & 0.5 & 0.6 & 1.5 \\
\hline
\end{tabular}

Tabla 3. Relación entre la toma de decisiones en temas de su propia Salud Sexual y Reproductiva (SSR) y los tipos de violencia física/sexual que sufre la entrevistada por parte del esposo

Tipos de agresión física/sexual que sufre la entrevistada por parte de su pareja
Número de decisiones que toma la entrevistada en temas de su propia SSR

\begin{tabular}{|c|c|c|c|}
\hline \multirow[b]{2}{*}{$\begin{array}{l}\text { 11pos de agresion fisica/sexual que sutre la entrevistada por parte } \\
\text { de su pareja }\end{array}$} & \\
\hline & $\begin{array}{l}\text { Mujeres que no } \\
\text { participan en } \\
\text { ninguna decisión }\end{array}$ & $\begin{array}{c}\text { Mujeres que } \\
\text { participan en } 1 \text { a } 2 \\
\text { decisiones }\end{array}$ & $\begin{array}{c}\text { Mujeres que } \\
\text { participan en } 3 \text { ó } \\
\text { más decisiones }\end{array}$ \\
\hline Empujó/hamaqueó & 2.4 & 5.4 & 4.6 \\
\hline La abofeteó & 4.9 & 4.1 & 2.7 \\
\hline Le dobló el brazo o le haló el cabello & 2.4 & 2.7 & 2.7 \\
\hline Le pegó con el puño o con algún objeto que le pudo lastimar & 5.0 & 4.0 & 2.3 \\
\hline La pateó, arrastró o le dio palizas & 0.1 & 1.1 & 0.9 \\
\hline Intentó quemarla o asfixiarla & 0.1 & 1.1 & 0.6 \\
\hline La atacó o amenazó con un cuchillo, pistola u otra arma & 2.5 & 0.5 & 0.8 \\
\hline La forzó físicamente a tener relaciones sexuales & 5.0 & 5.2 & 1.8 \\
\hline $\begin{array}{l}\text { La forzó físicamente a realizar cualquier acto sexual que ella no } \\
\text { quería }\end{array}$ & 4.9 & 2.1 & 0.4 \\
\hline La forzó con amenazas a realizar actos sexuales que ella no quiere & 7.3 & 1.7 & 0.4 \\
\hline
\end{tabular}

Fuentes: preparado por los autores con datos de ENASSER 2014-2015.

lud sexual y reproductiva (Indicador 3: Poder de Decisión más decisiones toman $(0.4 \%)(p=0.000)$. En cambio, en de la Mujer sobre su SSR) y como éste nivel de decisión el primer nivel de agresión, como son los empujones, los se asocia con la presencia o no de violencia física y/o se- porcentajes más altos de dan entre aquellas que más dexual que ella sufre por parte de su cónyuge (Tabla 3).

Entre las encuestadas que menos poder de decisión tiene sobre su propia salud sexual y reproductiva, el porcentaje más alto lo encontramos en el último renglón correspondiente a las mujeres que declararon ser víctimas de agresiones de tipo sexual, con $7.3 \%$ en aquellas mujeres sin ningún nivel de decisión, pero los porcentajes van disminuyendo (en este mismo tipo de agresión) entre las que toman de 1 a 2 decisiones (1.7\%) y aquellas que cisiones toman, $5.4 \%$ y $4.6 \%$ vs. $2.4 \%$ en aquellas que no toman ninguna decisión (Ver cuadro 3).

\section{DISCUSIÓN}


Flores y cols. - El empoderamiento de la mujer panameña.

o sexual que aquellas que no justificaron la violencia por ninguna razón. Este comportamiento de aceptación de la violencia de aquellas mujeres que más violencia conyugal sufren, está íntimamente ligado a creencias sociales y culturales que ven el ejercicio de la violencia masculina como algo "normal" [7]. Como señalan algunos autores muchas mujeres víctimas de la violencia conyugal, justifican a sus esposos quienes la agreden sin intención, motivados por razones ajenas o fuerzas externas a su voluntad [8]. Estas justificaciones de las mujeres están cargadas de sentimientos de culpa al considerar que el maltrato físico conyugal es algo "merecido" por el incumplimiento de las obligaciones del rol de género femenino $[9,10]$. Las mujeres con mayor participación en la toma de decisiones en el hogar presentan los porcentajes más altos en todos los tipos de violencia. Estos resultados parecen contradecir ese carácter protector que debe otorgar el empoderamiento femenino; sin embargo, nuestros resultados coinciden con algunos autores que hablan de esa asociación entre el proceso de empoderamiento de la mujer y el mayor riesgo de experimentar violencia por parte de su pareja. Se han encontrado evidencias que el empoderamiento de la mujer va acompañado de un exacerbamiento de la violencia de pareja, especialmente cuando las mujeres desafían el mando o autoridad de sus maridos $[11,12,13]$. Como mencionan Mason y Smith, el empoderamiento femenino es multidimensional, lo que contribuye que los vínculos entre los procesos de empoderamiento y la presencia de violencia conyugal sea una relación compleja de múltiples aristas, y así vemos, por ejemplo, como la capacidad de una mujer en las decisiones económicas del hogar no necesariamente coincide con su libertad de movimiento en la comunidad o la medida en que está sujeta a controles interpersonales coercitivos por su marido [11]. el mismo proceso de empoderamiento está conformado por múltiples elementos, y no siempre todos están presentes, como menciona Casi que, una mujer puede tener un alto nivel de empoderamiento en uno(s) de tales elementos, pero no en otros [13].

Aquellas mujeres con menor grado de participación en la toma de decisiones sobre su propia salud sexual y reproductiva son las que presentan los porcentajes más altos de agresiones, específicamente de tipo sexual. En términos generales, este tercer indicador de empoderamiento parece actuar como un factor de protección, disminuyendo los riesgos de violencia cuando adquieren ribetes de agresiones más agravadas como son el uso de armas blancas o de fuego o agresiones de tipo sexual. Datos similares fueron encontrados en un análisis secundario de una encuesta de hogares (ENDIREH 2003), donde se observa un conjunto de variables, entre ellas el que la mujer pueda decidir cuándo tener relaciones sexuales, que actúan como factores de protección para reducir el riesgo de violencia física, emocional, económica y sexual [13]. Pero cuando la mujer perciba que hay menos amenazas a su integridad física o sexual, como en el caso de los empujones, encontramos porcentajes más alto de violencia en aquellas que toman más decisiones sobre su salud sexual y reproductiva en comparación con las mujeres que menos decisiones toman.

\section{CONCLUSIONES}

Se encuentra una asociación negativa entre la toma de decisión en el hogar y la violencia que sufre la mujer, donde este elemento del empoderamiento no parece ofrecer un papel protector frente a la violencia.

Hay una asociación positiva entre el menor grado de violencia conyugal y mayores niveles de autonomía con respecto a una mayor capacidad para decidir sobre acciones concernientes a su propia salud sexual y reproductiva, como el negociar los términos de una relación sexual o el uso de métodos anticonceptivos.

Igualmente se encuentra una asociación positiva entre las mujeres que menos justifican la violencia de género y una menor vivencia de violencia conyugal.

Los tres elementos del empoderamiento femenino analizados en este trabajo se relacionan con la violencia física y sexual, que sufre la mujer por parte de su parte de su pareja sexual.

Todo esto resalta la importancia de trabajar en esas ideologías de género que persisten en nuestra sociedad, se deben usar todos los recursos disponibles de educación formal y no formal para la construcción de patrones culturales que persisten en la idiosincrasia, no solo de los hombres, sino también de las propias mujeres.

\section{REFERENCIAS}

[1] Ministerio Público, República de Panamá, Disponible en: https://ministeriopublico.gob.pa/wp-content/ uploads/2020/01/Informe-Estadístico-ViolenciaDoméstica-Enero-a-Diciembre-de-2019

[2] Organización Mundial de la Salud, Violencia inflingida por la Pareja, WHO 2013, Disponi-ble en: https:// apps.who.int/iris/bitstream/handle/10665/98816/ WHO RHR 12.36 spa.pdf;jsessionid

[3] Batliwala, Srilatha. "The Meaning of Women's Empowerment: New Concepts from Ac-tion". En Population Policies Reconsidered, coordinado por Gita Sen, Adrienne Germain y Lin-coln Chess. Cambridge: Harvard University Press, 1994.

[4] Garcia-Moreno C et al. Estudio multipaís de la OMS sobre salud de la mujer y violencia doméstica contra la mujer: primeros resultados sobre prevalencia, eventos relativos a la salud y respuestas de las mujeres a dicha violencia. Ginebra, Organización Mundial de la Salud, 2005)

[5] Ghuman, Sharon J. (2001). Employment, Autonomy and Violence against Women in India and Pakistan, University of Pennsylvania, Population Studies Center.

[6] Schuler, S.R.; Hashemi, Riley and Akhter, S. (1996). "Credit Programs, Patriarchy and Men's Violence against Women in Rural Bangladesh", Social Science and Medicine 43: 1792-1742. 
[7] Heise L, Pitanguy J, Germain A. Violencia contra la mujer: la carga oculta sobre la salud. Washington D.C.: OPS, 1994.

[8] Eisikovits Z, Winstok Z, Fishman G. The first Israeli National Survey on Domestic Vio-lence. Violence Against Women 2004: 729-748.

[9] Güezmes A, Palomino N, Ramos M. Violencia sexual y física contra las mujeres en el Perú. Estudio multicéntrico de la OMS sobre la violencia de pareja y la salud de las mujeres. Lima: OMS /Centro de la Mujer Peruana Flora Tristán /Universidad Peruana Cayetano Heredia, 2002.

[10] Carolina Agoff, Ari Rajsbaum, Cristina Herrera. Perspectivas de las mujeres maltratadas sobre la violencia de pareja en México. Salud Pública de México vol 48 supl.2 Cuernavaca, Méxi-co 2006.
[11] Mason, K. y Smith, H. (2003). Women's Empowerment and Social Context: Results from five Asian Countries, University of Pennsylvania. https://www.semantic scholar.org/paper/WOMEN'S-EMPOWERMENTAND-SOCIAL-CONTEXT\%3A-RESULTS-MasonSmith/68423faadedb45524c55263554bea269d2f4fb15 (12) Sen, Gita. Empoderamiento de las mujeres y procesos demográficos: más allá de EI Cairo Oxford University Press. 2000 https://iucat.iu.edu/iuk/4941041

[13] Cacique, I. Multiplicidad del vínculo entre empoderamiento de la mujer y violencia de género. http://biblioteca2.ucab.edu.ve/iies/bases/iies/texto/ CASIQUE_IR_2008.PDF.pdf 